\title{
Erratum to: Oxytocin Signaling in Pain: Cellular, Circuit, System, and Behavioral Levels
}

Pierrick Poisbeau, Valery Grinevich, and Alexandre Charlet

\author{
Erratum to: \\ Chapter "Oxytocin Signaling in Pain: \\ Cellular, Circuit, System, \\ and Behavioral Levels" in: Curr Topics \\ Behav Neurosci, \\ DOI 10.1007/7854_2017_14
}

As per the request of volume editor, the affiliation of Dr. Valery Grinevich is updated as below in the chapter opener page.

Schaller Research Group on Neuropeptides, German Cancer Research Center, University of Heidelberg, Heidelberg, BW, Germany

Central Institute of Mental Health, University of Heidelberg, Mannheim, BW, Germany

\footnotetext{
The updated online version for this chapter can be found at DOI 10.1007/7854_2017_14
} 